\title{
Slip, concentration fluctuations and flow instability in sheared polymer solutions
}

\author{
William B. Black*and Michael D. Graham ${ }^{\dagger}$ \\ Department of Chemical Engineering and Rheology Research Center \\ University of Wisconsin-Madison, Madison, WI 53706-1691
}

October 24, 2018

\begin{abstract}
Recent experiments suggest that shear-enhanced growth of concentration fluctuations in polymer solutions is strongly influenced by solid boundaries. We analyze the dynamics of a model of a sheared polymer solution, accounting for the effect of stress variations on mass flux and for wall-slip. If (and only if) these effects are present, the flow exhibits a boundary-localized instability consistent with experimental observations. Even in the absence of flow instability, thermally-driven concentration fluctuations are significantly enhanced near the boundary.

PACS numbers: $61.25 . \mathrm{Hq}$, 83.50.Lh
\end{abstract}

The local composition of polymer solutions and blends can be significantly affected by flow. In particular, shear-enhanced concentration fluctuations in semidilute polystyrene (PS) solutions have been observed via light-scattering and microscopy by a number of groups (1); 2; 3; 4; 5; 6; 7; 8). A purely hydrodynamic mechanism for this phenomenon, in an unbounded flow domain, was proposed initially by Helfand and Fredrickson (HF) (9) and later extended by several authors (10; 11; 12; 13; 14). This mechanism is based on the idea that variations in polymer stress can drive a flux of polymer molecules - indeed, several authors (15; 12; 13; 16) have developed simple, two-fluid models which demonstrate quite generally that the polymer flux in a solution, $\boldsymbol{j}$, has the general form $\boldsymbol{j}=-D \boldsymbol{\nabla} n+\left(D / k_{B} T\right) \boldsymbol{\nabla} \cdot \boldsymbol{\tau}$, where $n$ is the polymer number density, $\boldsymbol{\tau}$ is the polymer extra stress tensor, $D$ is the polymer gradient diffusivity, $k_{B}$ is the Boltzmann constant, and $T$ is the temperature. Furthermore, a detailed kinetic theory derivation for a dilute solution (17) leads to a very similar expression for the flux. A scattering peak is predicted to arise when the diffusion and relaxation times are equal, which occurs at a wavelength of $2 \pi / k \approx \sqrt{D \lambda}$ which is, not coincidentally, the only inherent length scale in the problem.

This length scale is observed in light scattering experiments. Hashimoto and coworkers (1; 2; 5; 8) have performed a series of experiments with PS dissolved in dioctylphthalate (DOP)

*Present address: 3M Corporate Process Technology Center, St. Paul, MN, 55144

${ }^{\dagger}$ Corresponding author. E-mail: graham@engr.wisc.edu 
and have measured the scattering intensity by illuminating along the shear-gradient direction (the $y$-direction of Fig. 1). They observed a "butterfly" scattering pattern indicative of flow structures with wave vectors roughly aligned in the flow direction. The wavenumbers obtained from the patterns were on the order of $k \approx 0.6 \mu \mathrm{m}^{-1}$ giving a length scale for the fluctuations of approximately $10 \mu \mathrm{m}$. Estimating the relaxation time as the reciprocal of the shear rate for the onset of shear thinning (8) and using the diffusivity measured by $\mathrm{Wu}$ et al. (3) for a similar system gives a predicted wavenumber of $k \approx 0.7 \mu \mathrm{m}^{-1}$, which is close to the measured value. This length scale was also observed by $\mathrm{Wu}$ et al. (3), who measured the scattering intensity by illuminating along the vorticity direction (the $z$-direction in Fig. 1). At low shear rates, the scattering peak was located at $k \approx 10 \mu \mathrm{m}^{-1}$, which is in good agreement with their predicted value of $9.4 \mu \mathrm{m}^{-1}$.

Even though both of these experiments show peaks at the requisite length scale, qualitative differences exist in the shear rate dependence of the results. In particular, Hashimoto and coworkers, who measured the intensity essentially averaged over a volume containing both near-surface regions, found that the intensity increased markedly above a critical shear rate, which was roughly the reciprocal of the polymer relaxation time (an approximately 100fold increase in scattering for a 10-fold increase in shear rate). Conversely, Wu et al., whose measurements sample only the bulk, not the near-surface regions, found a much smaller increase in intensity as the shear rate was increased, approximately an 11-fold increase in scattering intensity for a 25 -fold increase in shear rate.

A recent experimental study by Mhetar and Archer (18) has demonstrated several novel features of shear enhanced concentration fluctuations in entangled polymer solutions. They studied semidilute solutions of PS in diethylphthalate (DEP), to which tracer particles were added to measure the velocity profile, using a planar Couette cell with (high energy) silica walls. In this situation, wall-slip occurs primarily due to the disentanglement of bulk chains from those adsorbed to the walls, rather than to the formation of a depletion layer, as arises in dilute solutions $(19 ; 20)$. Two features of the flow of these solutions stand out. First, no turbidity was observed at low shear stresses where slip was weak; enhanced concentration fluctuations were observed, however, at large shear stresses, where slip was significant. The length scale for the fluctuations was observed to be $\approx 10 \mu \mathrm{m}$, as was the extrapolation, or slip, length measured for these solutions. Second, the concentration fluctuation enhancement began near the boundaries and modification of the surface to increase slip delayed the onset on fluctuations to much larger shear stresses. These observations strongly suggest that slip plays a role in the formation and development of these fluctuations.

Taken together, experiments suggest that the HF mechanism, while being able to explain bulk behavior, only partially accounts for the physics leading to enhancement of fluctuations near surfaces, a conclusion that can be put into perspective by consideration of the fluid dynamics of polymer flows near boundaries. Gorodtsov and Leonov (22) analyzed the plane Couette flow of an upper convected Maxwell (UCM) fluid (a model of a melt) and found that, while the flow is always linearly stable, the most slowly decaying modes are localized near the bounding surfaces. As a result, we might expect the formation of boundary localized structures due to random concentration fluctuations in the polymer solution. In addition, slip has been shown to lead to boundary-localized flow instability in polymeric simple shear flows under certain conditions (23; 24; 25). This suggests that boundary-localized concentration fluctuation dynamics can appear by analogous routes. Our goal here is to analyze the 
coupling between concentration, stress, and slip in bounded shear flow, to elucidate the effects of boundaries on the dynamics.

We consider flow in the plane Couette geometry shown in Fig. 11. To describe the behavior of the solution, we use the kinetic theory model derived by Beris and Mavrantzas (26), for a solution of Hookean dumbbells, and written in terms of the conformation tensor, $\boldsymbol{\alpha}=\langle\boldsymbol{Q} \boldsymbol{Q}\rangle$, which is the second moment of the orientation vector of the dumbbells, $\boldsymbol{Q}$. Nondimensionalizing stresses with $n_{0}^{*} k_{B} T$, where $n_{0}^{*}$ is the average concentration of the solution, lengths with the characteristic length $\sqrt{D \lambda}$, velocities with $\dot{\gamma}^{*} \sqrt{D \lambda}$, time with $\lambda$, and concentration with $n_{0}^{*}$ gives the following model equations:

$$
\begin{array}{r}
\frac{\partial \boldsymbol{\alpha}}{\partial t}+\boldsymbol{v} \cdot \boldsymbol{\nabla} \boldsymbol{\alpha}-\boldsymbol{\alpha} \cdot \boldsymbol{\nabla} \boldsymbol{v}-(\boldsymbol{\nabla} \boldsymbol{v})^{T} \cdot \boldsymbol{\alpha}+\boldsymbol{\alpha}-n \boldsymbol{\delta}=\nabla^{2} \boldsymbol{\alpha} \\
\frac{\partial n}{\partial t}+\boldsymbol{v} \cdot \boldsymbol{\nabla} n=-\boldsymbol{\nabla} \cdot(-\boldsymbol{\nabla} n+\boldsymbol{\nabla} \cdot \boldsymbol{\tau}), \\
-\boldsymbol{\nabla} p+\boldsymbol{\nabla} \cdot \boldsymbol{\tau}+S \boldsymbol{\nabla}^{2} \boldsymbol{v}=0, \\
\boldsymbol{\nabla} \cdot \boldsymbol{v}=0,
\end{array}
$$

where $\boldsymbol{v}$ is the velocity, $S$ is the ratio of the solvent to polymer viscosities, $n$ is the number density of dumbbells, $\boldsymbol{\tau}=\boldsymbol{\alpha}-n \boldsymbol{\delta}$ is the polymer extra stress and $p$ is the pressure. In presenting the model, we have suppressed any dependencies of properties on concentration in the analysis below we only consider small perturbations from spatially uniform stress and concentration fields, in which case the terms that reflect these dependencies do not appear. Eq. 1 describes the evolution of polymer conformation and is a generalization of the upper convected Maxwell (or Oldroyd-B) model (27) to include spatial diffusion of conformation (on the RHS). In most cases, this term is negligible (26), but here, the flow structures we are interested in have a length scale set by the diffusivity. Eq. 2 is the conservation equation for the dumbbells, explicitly showing the dependence of the polymer flux on stress variations. If diffusion of the polymer molecules is neglected, the right-hand sides of Eqs. 1 and 2 vanish, recovering the Maxwell model. Eqs. 3 and 1 describe conservation of momentum and mass. Other phenomenologically motivated models for such systems are virtually identical to this one, but do not generally include the conformational diffusion term (9; 10; 12; 13; 14), the primary difference being the use of the polymer phase velocity rather than the massaverage velocity in the deformation terms of the equation for polymer conformation - our preliminary results with such a full two-fluid model yield qualitatively identical results to those presented below. Furthermore, all earlier works have restricted themselves to bulk behavior and $W e<1$.

Because of the high energy surfaces in Mhetar and Archer's experiments, it is reasonable to assume that the adsorbed chains do not desorb to a significant degree in flow, and that no additional bulk chains adsorb. Therefore, we apply a no-flux boundary condition for the polymer concentration: $\boldsymbol{n} \cdot \boldsymbol{j}=\partial n / \partial y-\partial \tau_{y x} / \partial x-\partial \tau_{y y} / \partial y-\partial \tau_{z y} / \partial z=0$, where $\boldsymbol{n}$ is the unit normal on the boundary. Inclusion of the diffusion term in the constitutive equation necessitates the specification of boundary conditions for the conformation tensor. For the same reason as above, we assume that there are no sources or sinks for polymer conformation at the walls, giving a no-flux boundary condition for the conformation, $\partial \boldsymbol{\alpha} / \partial y=0$. We have also performed computations using the classical UCM equation as the boundary condition 
for $\boldsymbol{\alpha}(28)$ and found no qualitative difference in the results.

The model is completed by specifying the slip boundary condition at the interface, which crudely reflects the interaction between the bulk chains and those attached to the wall. We use here the simplest nontrivial model, the Navier slip boundary condition $u_{s}=\epsilon \tau_{y x}(=b=$ $b^{*} / \sqrt{D \lambda}$ at steady state); $\epsilon$ is given by $b /$ We.

We have studied the dynamics of this model primarily by linear stability analysis of the plane Couette base state, given by $\overline{\boldsymbol{a}}=\left(\bar{v}_{x}, \bar{v}_{y}, \bar{v}_{z}, \bar{\tau}_{x x}, \bar{\tau}_{y x}, \bar{\tau}_{z x}, \bar{\tau}_{y y}, \bar{\tau}_{z y}, \bar{\tau}_{z z}, \bar{n}, \bar{p}\right)$ $=\left(W e(y+b), 0,0,2 W e^{2}, W e, 0,0,0,0,1,1\right)$, where $\boldsymbol{\alpha}$ has been eliminated in favor of $\boldsymbol{\tau}$. Small perturbations are added to this base solution, i. e. $\boldsymbol{a}=\overline{\boldsymbol{a}}(y)+\tilde{\boldsymbol{a}}(x, y, z, t)$, and only the terms linear in the perturbation are retained in the model equations. The perturbations take the normal mode form: $\tilde{\boldsymbol{a}}(x, y, z, t)=\hat{\boldsymbol{a}}(y) e^{i \boldsymbol{k} \cdot \boldsymbol{x}} e^{-i \sigma t}+$ c.c., where $\boldsymbol{k}=\left(k_{x}, 0, k_{z}\right)$. This formulation yields a generalized eigenvalue problem for the eigenvalues $\{\sigma\}$ with associated eigenfunctions $\{\hat{\boldsymbol{a}}(y)\}$. If $\operatorname{Im}(\sigma)>0$ then infinitesimal disturbances grow and the flow is unstable.

Prior work (24; 25) with the UCM model and preliminary work with the present model have shown that the eigenfunctions of the discrete spectrum are localized near the boundaries. Furthermore, in experiments, $\sqrt{D \lambda} \ll l^{*}$. Therefore, we solve the eigenvalue problem on a semi-infinite domain (SID). We map $y \in[0, \infty)$ onto the computational domain, $\xi \in[-1,1]$, using the mapping $y=0.1(1+\xi) /(1-\xi)$, and then perform Chebyshev collocation on the mapped system (29), using $N+1$ collocation points. The eigenvalue problem is solved using a public domain routine (30).

To begin the discussion of the results, we note that if the possibility of concentration fluctuations is suppressed in the model (by setting $D$ to zero, thus eliminating the RHS of eqs. 11 and 2), plane shear flow with either the no-slip (22) or Navier slip (31; 24; 25) boundary condition is stable for all Weissenberg numbers. In addition, we have found that if concentration variations are permitted but slip is not (by setting $b=0$ ), the flow is also stable. In contrast, if slip and concentration variations are allowed, the flow can become unstable when $W e$ exceeds a critical value $W e_{c}-$ a new class of polymer flow instabilities arises. Fig. 2 shows neutral curves, plotted as $W e_{c}$ vs. $k_{x}$ for two dimensional disturbances, i.e. $k_{z}=0$, with $b$ fixed. For $b=O(1)$, the critical wavenumber is $O(1)$, so that the critical length scale for the instability is $\sqrt{D \lambda}$. We have also examined stability with respect to three dimensional disturbances $\left(k_{z} \neq 0\right)$, but find that two dimensional disturbances are always the most dangerous. At low wavenumbers (those most easily observed by microscopy), $W e_{c}$ first decreases and then increases with increasing $b$ - the increasing section of the curve is in agreement with the observations of Mhetar and Archer (18) that treating the surface to increase slip delayed the onset of fluctuations. Note that there is a transition from one mode of instability to another, leading to multiple minima in the neutral curve, as most clearly seen in the curve for $b=2$. For larger values of $b$, the neutral curves collapse toward a single curve. Similar results are found for larger values of $S$, but the critical Weissenberg numbers are much lower, $W e_{c} \approx 2$ for $S \gtrsim 1$. (However, for $S=O(1)$, the solution would typically be too dilute for slip to be a realistic boundary condition.)

Fig. 3 shows a density plot of a typical destabilizing concentration perturbation. Near the boundary, the local wavevector has the same orientation as that predicted by the HF mechanism (9; 13). In agreement with the observations of Mhetar and Archer (18), pertur- 
bations are localized near the surfaces and are observed at large We. As a result, we would expect scattering to be more pronounced in the boundary regions than in the bulk.

We have also investigated the dynamics of Brownian fluctuations in this model. Ji and Helfand (13) have demonstrated that the velocity is a fast variable and therefore, since introduction of concentration fluctuations into this system results in adding fluctuations directly to the stress, we only incorporate the random forcing into the polymer mass balance. This is carried out by adding a term $\nabla \cdot \boldsymbol{w}$ to Eq. 2, where $\boldsymbol{w}$ is the random contribution to the polymer flux and satisfies $\left\langle\boldsymbol{w}(\boldsymbol{x}, t) \boldsymbol{w}\left(\boldsymbol{x}^{\prime}, t^{\prime}\right)\right\rangle=\left[\left(2 n_{0}^{*}(D \lambda)^{3 / 2}\right)\right] \boldsymbol{\delta} \delta\left(\boldsymbol{x}-\boldsymbol{x}^{\prime}\right) \delta\left(t-t^{\prime}\right)$ (32). To conserve mass, we require $w_{y}=0$ on $y=0,1$. We follow the common procedure (9; 10; 12; 13) of assuming the noise is small and contributes only to the linearized equations. Again, Chebyshev collocation is used for the spatial discretization in the $y$-direction with a Fourier transform in the $x$-direction, but because the collocation points are not uniformly distributed, the random forcing is weighted so that it is uniform in magnitude throughout the domain. The resulting system of equations was time-integrated on a bounded domain using an implicit Euler scheme with the top plate located at $l^{*}=100 \sqrt{D \lambda}$. Results are collected only after the initial transient has decayed and fluctuation effects dominate.

To quantify the response as a function of time, we compute the concentration correlation function for a given $x$-wavenumber $k_{x}:\left\langle\tilde{n}\left(\boldsymbol{x} ; k_{x}\right) \tilde{n}\left(\boldsymbol{x}^{\prime} ; k_{x}\right)\right\rangle=2 \operatorname{Re}\left\langle\hat{n}\left(y ; k_{x}\right) \hat{n}^{*}\left(y^{\prime} ; k_{x}\right)\right\rangle \cos \left(k_{x}(x-\right.$ $\left.\left.x^{\prime}\right)\right)-2 \operatorname{Im}\left\langle\hat{n}\left(y ; k_{x}\right) \hat{n}^{*}\left(y^{\prime} ; k_{x}\right)\right\rangle \sin \left(k_{x}\left(x-x^{\prime}\right)\right)$. Fluctuations are strongly correlated near the surfaces, even at equilibrium $(W e=0)$. At equilibrium, $\operatorname{Im}\left\langle\tilde{n}(y) \tilde{n}^{*}\left(y^{\prime}\right)\right\rangle$ is zero, but for nonzero We, it assumes values consistent with enhanced fluctuations whose wave vectors are rotated from the flow direction into the first quadrant, while the magnitude of the real part remains essentially unchanged. A snapshot of a typical concentration profile is shown in Fig. 1 for $W e=1, k_{x}=1, b=1$ and the orientation, which is again consistent with the bulk $\mathrm{HF}$ mechanism, is clear. Hence, fluctuations form near the surface by mechanisms analogous to those in the bulk, but are enhanced because of the vicinity to the no-flux boundary. Results for the no-slip case are virtually identical - the response of the flow to Brownian noise is quite insensitive to the presence of slip. Therefore, even in the absence of flow instability, (i.e. when $W e \lesssim O(1)$ and/or slip is absent), the near surface regions may make a nontrivial contribution to the scattering signal, particularly its anisotropy under flow. The insensitivity to slip is consistent with what is known about the eigenspectra of viscoelastic flows: whether or not there is slip, the slowest decaying eigenmodes are localized near solid boundaries.

These results clearly suggest that the bounding surfaces play an important role in the formation of enhanced concentration fluctuations in sheared polymer solutions. In the context of the present model, slip leads to a new class of viscoelastic flow instabilities which result from the interaction of slip with stress and concentration, and even in the absence of slip, Brownian fluctuations are selectively enhanced near the surfaces. These results are consistent with experimental observations and underscore two points regarding the flow behavior of polymeric liquids: (1) the distinctness and importance of the dynamics of flowing polymers near boundaries, even at the continuum level, and (2) the importance of couplings between various phenomena for the dynamics and stability of these flows.

The authors gratefully acknowledge the National Science Foundation and the Nontenured faculty grant program of the 3M Company for supporting this work. 


\section{References}

[1] T. Hashimoto and K. Fujioka, J. Phys. Soc. Jpn. 60, 356 (1991).

[2] T. Hashimoto and T. Kume, J. Phys. Soc. Jpn. 61, 1839 (1992).

[3] X.-L. Wu, D. J. Pine, and P. K. Dixon, Phys. Rev. Lett. 66, 2408 (1991).

[4] P. K. Dixon, D. J. Pine, and X. Wu, Phys. Rev. Lett. 68, 2239 (1992).

[5] E. Moses, T. Kume, and T. Hashimoto, Phys. Rev. Lett. 72, 2037 (1994).

[6] D. Wirtz, Phys. Rev. E 50, 1755 (1994).

[7] H. Murase et al., Macromol. Symp. 104, 159 (1996).

[8] T. Kume, T. Hashimoto, T. Takahashi, and G. G. Fuller, Macromolecules 30, 7232 (1997).

[9] E. Helfand and G. H. Fredrickson, Phys. Rev. Lett. 62, 2468 (1989).

[10] S. T. Milner, Phys. Rev. Lett. 66, 1477 (1991).

[11] M. Doi and A. Onuki, J. Phys. II 2, 1631 (1992).

[12] S. T. Milner, Phys. Rev. E 48, 3674 (1993).

[13] H. Ji and E. Helfand, Macromolecules 28, 3869 (1995).

[14] T. Sun, A. C. Balazs, and D. Jasnow, Rhys. Rev. E 55, 6344 (1997).

[15] A. Onuki, J. Phys. Soc. Japan 59, 3423 (1990).

[16] V. G. Mavrantzas and A. N. Beris, Phys. Rev. Lett. 69, 273 (1992).

[17] C. F. Curtiss and R. B. Bird, J. Chem. Phys. 111, 10362 (1999).

[18] V. Mhetar and L. A. Archer, Macromolecules 31, 6639 (1998).

[19] M. D. Graham Chaos 9, 154 (1999).

[20] D. Aussere, H. Hervet and F. Rondelez, Macromolecules 149, 85 (1982).

[21] A. Onuki, Physica A 204, 499 (1994).

[22] V. A. Gorodtsov and A. I. Leonov, J. Appl. Math. Mech. 31, 310 (1967).

[23] M. Renardy, J. Non-Newtonian Fluid Mech. 35, 73 (1990).

[24] W. B. Black and M. D. Graham, Phys. Rev. Lett. 77, 956 (1996).

[25] W. B. Black and M. D. Graham, Phys. Fluids 11, 1749 (1999).

[26] A. N. Beris and V. G. Mavrantzas, J. Rheol. 38, 1235 (1994).

[27] R. B. Bird, C. F. Curtiss, R. C. Armstrong, and O. Hassager, Dynamics of Polymeric Liquids (Wiley - Interscience, New York, 1987), Vol. 2.

[28] R. Sureshkumar and A. N. Beris, J. Non-Newtonian Fluid Mech. 60, 53 (1995).

[29] C. Canuto, M. Y. Hussaini, A. Quarteroni, and T. A. Zang, Spectral Methods in Fluid Dynamics (Springer-Verlag, Berlin, 1988).

[30] B. S. Garbow, ACM Transactions on Mathematical Software 4, 404 (1978). 
[31] J. R. A. Pearson and C. J. S. Petrie, in Polymer Systems: Deformation and Flow, Proceedings of the 1966 Annual Conference of the British Society of Rheology, edited by R. E. Wetton and R. W. Whorlow (Macmillan, London, 1968), pp. 163-187.

[32] C. W. Gardiner, Handbook of Stochastic Methods (Springer-Verlag, Berlin, 1985). 


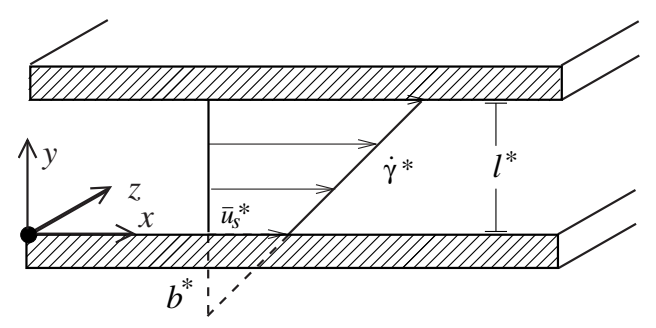

Figure 1: Plane Couette geometry showing slip between the solution and solid surface. The flow is in the $x$-direction, $y$ is the gradient direction and $z$ is the vorticity or neutral direction. $l^{*}$ is the gap width, $u_{s}^{*}$ is the slip velocity, $\dot{\gamma}^{*}$ is the true shear rate, and $b^{*} \equiv u_{s}^{*} / \dot{\gamma}^{*}$ is the steady-state extrapolation length. The asterisks (here and in the text) denote dimensional quantities.

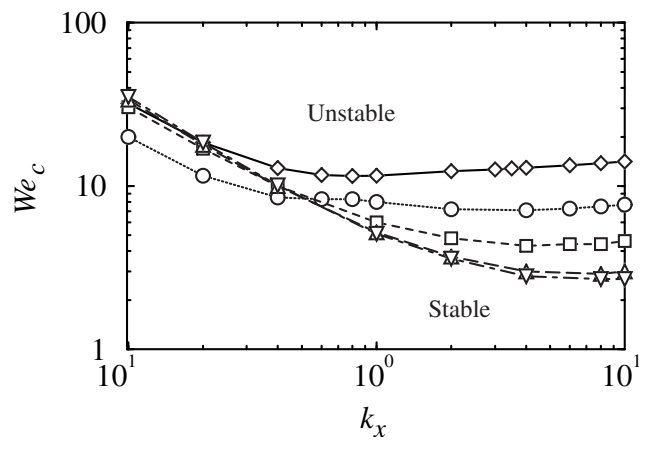

Figure 2: Neutral curves for $k_{z}=0, S=10^{-2}, N=96$, and various values of the extrapolation length. $\diamond-b=1, \bigcirc-b=2, \square-b=4, \triangle-b=8$, and $\nabla-b=10$. 


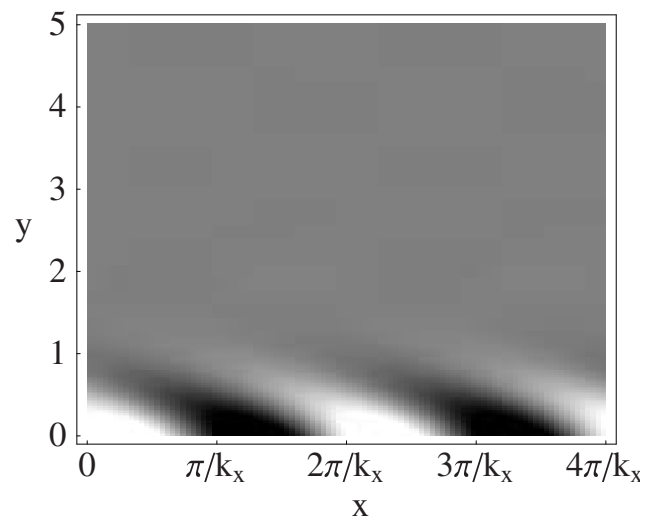

Figure 3: Concentration component of the unstable eigenfunction; $k_{x}=0.4, b=10$, We $=10$, $S=0.01, N=96$.

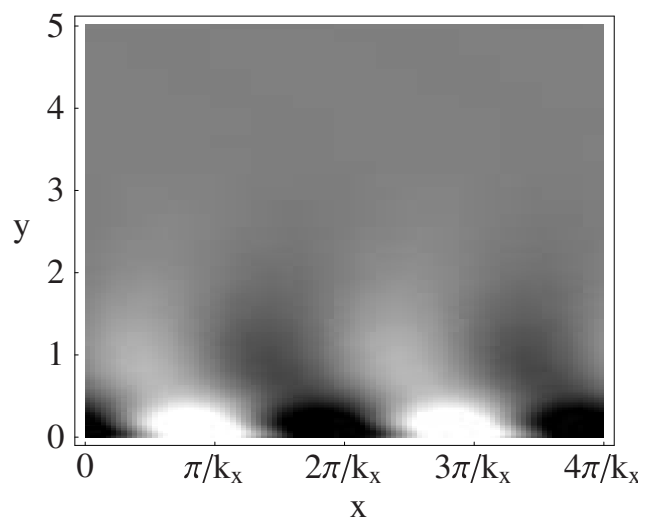

Figure 4: Typical snapshot of a concentration profile driven by random fluctuations. The parameters are: $W e=1, k_{x}=1, b=1, S=0.01, N=192$. 